\title{
Open Access to Academic Research
}

\section{It is June and as we do every year, ACM celebrates the extraordinary accomplishments and contributions of some of our colleagues in computer science and information}

technology. This year, we honor Sir Tim Berners-Lee as the recipient of ACM's most prestigious 2016 A.M. Turing Award. The impact of the World Wide Web has steadily increased since it was first introduced in late 1991 and stands as a remarkable infrastructure, enhancing the value of the Internet, datacenters, smartphones, and all manner of other programmable systems.

This year also marks the first time the new ACM Prize in Computing is awarded-the inaugural recipient is Alexei A. Efros, for his groundbreaking, data-driven approaches to computer graphics and computer vision. Many other deserving recipients will be feted at the annual ACM Gala in San Francisco on June 24 and I hope to see many of you there to celebrate the achievements of our colleagues.

I recently spent a half-day with a group of government agency, publishing industry, and academic institutional representatives to discuss key considerations leading to improved access to academic research results, associated data, and analytic software. The sponsoring organization is the Open Scholarship Initiative (osinitiative.org) and its primary goal is accessibility of research and scholarly output. This was not necessarily about free access as much as making things easily discoverable and accessible.

The world of scientific and academic publishing has grown over time and this is not surprising. The number of narrowly focused academic publications is increasing, partly driven by business models of profit and non- profit institutions and partly by the "publish or perish" dynamic in the academic world. Library budgets have not kept up with the cost of increasing numbers of publications. Changing this dynamic may necessitate revising the metrics of value: favoring quality over quantity. Academic tenure decisions often seem to turn on quantity and perhaps that must change.

Reproducibility of reported research is an important trend and is aided by funding-agency requirements for the preservation of research data, associated metadata, software and equipment documentation, as well as reported analytic results. This is the essence of the scientific method and is a laudable goal. The U.S. National Science Foundation sponsors the Research Data Alliance that undertakes to achieve this objective. Preservation of software and its execution environments is a topic about which I have written more than once in this column so I will simply reiterate here the challenge and importance of achieving this objective.

One of the challenges associated with data and publication preservation is the creation of business models that can sustain long-duration archives over decades, if not centuries. Present publication business models often sustain significant portions of the operating costs for academic organizations. For many research publishers, all of their operating costs must be covered this way. Ironically, researchers have often noted that academic colleagues at little direct cost to publishers undertake the editing and review functions of most scientific journals. This has led some institutions to consider the formation of digital publication processes staffed by volunteer editors and reviewers. It should be noted, however, that considerable infrastructure must be maintained to ensure access to digital content over long periods of time and that, too, has an underlying cost.

In a recent National Academy of Engineering Spring Symposium, one of the speakers, Bret Victor, offered a working example of a modern-day digital publication that was far from static. Indeed, the tables and charts were fully interactive. Readers could alter parameters to see how the results might look under varied conditions. This kind of composite publication might indeed become the forerunner of a mechanism for reproducibility, especially if the researcher's tools might be accessed through the publication to test new hypotheses or to feed new data into the system.

In the ensuing discussions, it seemed clear the participants who might have been expected to be at odds on the economics of research publication were in fact open to exploring new ways to ensure increasingly open access to research results and data. I was reminded of the success of the Human Genome Project and the role that publishers played: if your papers were to be published, you had to agree to put your discovered genetic sequences into one of three international human genome databases. The resulting sharing of this key information accelerated our understanding of the human genome and its implications.

Vinton G. Cerf is vice president and Chief Internet Evangelist at Google. He served as ACM president from 2012-2014.

Copyright held by author. 